\title{
German Guidelines on Screening, Diagnosis and Treatment of Alcohol Use Disorders
}

\author{
Karl Mann ${ }^{a} \quad$ Anil Batra $^{b} \quad$ Mira Fauth-Bühler ${ }^{a}$ \\ Eva Hoch ${ }^{a, c}$ and the Guideline Group

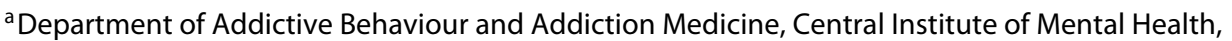 \\ Medical Faculty Mannheim/Heidelberg University, Mannheim, ${ }^{\text {b}}$ Department of Psychiatry and \\ Psychotherapy, University Hospital Tuebingen, Tuebingen, and ' Department of Psychiatry and \\ Psychotherapy, Ludwig-Maximilians-University Munich, Munich, Germany
}

\section{Keywords}

Guideline · Screening · Diagnoses · Treatment · Alcohol use disorders · Evidence base

\begin{abstract}
Evidence-based strategies for screening, diagnosing and treating alcohol use disorders (AUD) are instrumental in the early and better management of individuals at risk for or suffering from AUD. However, existing guidelines vary and may be biased by conflicts of interests. Unbiased recommendations can be achieved only if sufficient detail is provided on the composition and representativeness of author groups, methodological rigor, handling of potential conflicts of interest and financing. This paper presents the first evidencebased guidelines for AUD from German-speaking countries. These guidelines are based on the work of delegates from a representative sample of 46 scientific societies (mostly medical) from Austria, Germany and Switzerland dealing with AUD. It also included patients and relatives. Recommendations were derived from a standardised hierarchical process involving quality controls drawn from existing guidelines, de novo literature searches and/or expert experience. Potential conflicts of interest were assessed yearly and led to exclusion from voting in specific areas. An overall cost of more than
\end{abstract}

$400,000 €$ (for alcohol and tobacco guidelines) were exclusively covered by the participating societies and academic institutions. More than 100 recommendations on screening, diagnostics and treatment of AUD are outlined in this paper, and their scientific background is given in the online supplementary material. Tables of aggregated study synopses (in English) and the full version of guidelines (in German) are available (see "Links").

(c) 2017 S. Karger AG, Basel

\section{Introduction}

According to the actual estimates, there are currently more than 2 million adults ( 1.8 million in the age range of 18-64 years) in Germany who fulfill the criteria for alcohol dependence [1]. Another 2 million suffer from

The European Federation of Addiction Societies (EUFAS) supports the German Guidelines on "Screening, Diagnosis and Treatment of Alcohol Use Disorders." EUFAS recommends the guidelines to be considered in its member societies in Europe and beyond. Along with the guidelines of the National Institute for Health and Clinical Excellence (NICE) in the United Kingdom, they provide an evidence-based approach for handling alcohol use disorders.

\section{KARGER}

(C) 2017 S. Karger AG, Basel

E-Mail karger@karger.com

www.karger.com/ear
Prof. em. Karl Mann, MD

Central Institute of Mental Health

Senior Professor, Medical Faculty Mannheim/Heidelberg University

Square J5, DE-68159 Mannheim (Germany)

E-Mail karl.mann@zi-mannheim.de 
harmful use of alcohol. Nearly 74,000 people die annually due to alcohol-related causes [2]. Alcohol is considered a major risk factor for various somatic diseases and can have severe psychosocial impacts $[3,4]$. In contrast to other mental disorders such as depression, the general public considers alcohol use disorders (AUD) much less of a problem that needs attention and treatment. Individuals with problematic alcohol intake tend to ignore, deny or trivialise their alcohol-related problems, a behaviour that is considered part of their disorder. Medical treatment often focuses on comorbidities and secondary diseases. Failure to recognise AUD as causal in these individuals hinders health care professionals to adequately treat these patients.

Estimation models suggest that in Germany 2,000 lives could be saved per year if the rates of patients in psychoand/or pharmacological treatment increased from 10 to $40 \%$ [5]. To address the goal of involving more patients, we developed the current guideline for AUD to screen, diagnose and treat individuals suffering from AUD. The guideline conforms to the highest quality standards of the association of scientific medical societies (AWMF) [6] in Germany and constitutes the first such guideline in German-speaking countries. The recommendations summarised in the following are based on a systematic literature search. A formal consensus was used to adopt the recommendations.

The guideline is aimed at improving screening, diagnosis and treatment of individuals with at-risk consumption of alcohol, harmful alcohol use or alcohol dependence [7]. While the primary endpoint in many studies is the reduction of alcohol consumption, abstinence from alcohol remains an important goal especially for dependent individuals. Systematically developed, the guideline serves as a basis for healthcare professionals and individuals concerned and their relatives. The systematic search considers studies which were published until the end of 2012. Results from studies published until early 2014 could be considered, but in this case of an "unsystematic search" only Clinical Consent Point (CCP) recommendations were possible according to AWMF [6]. The guideline fosters the use of the most effective interventions and helps to point out procedures and treatments with no or minimal evidence. It is important to differentiate between a treatment method which receives low evidence grades due to the lack of studies or due to a failure to prove effectiveness (and hence should be recommended as inefficient or even harmful for the patient).

The guideline should serve as a basis for decision making for the following groups of people:
- Adults with at-risk consumption of alcohol, harmful use or alcohol dependence, and specific patient groups with special needs (children, adolescents, pregnant women, and women, elderly people) and individuals concerned with somatic and psychiatric comorbidities and secondary diseases, and their relatives.

- Professionals (general practitioners, psychiatrists, psychotherapists, psychologists, social workers, social education workers, nurses, occupational therapists, staff in other institutions, legal advisors and others working in the social care system).

- Other persons and policy makers in the health and social system, who offer or organise support for people with psychological problems and psychiatric diseases. It is of great importance to recognise and treat risky alcohol use, harmful alcohol use or alcohol dependence in a broad spectrum of settings and care areas. Screenings, motivation and early interventions can, for example, be applied in the primary healthcare setting (general practitioners, general hospitals and emergency facilities) and in work and training places. In addition, a variety of highly specialised healthcare areas exist for individuals with AUD. Examples are detoxifications and qualified withdrawal treatments (QWT).

\section{Methods}

Systematic Search, Selection and Evaluation of

Scientific Evidences

The guideline "Screening, Diagnosis and Treatment of Alcohol Use Disorders" was developed between 2010 and 2015 following an iterative, hierarchical search process [6].

In a first step, the content and scope of the guideline was defined. Questions of high clinical relevance for the healthcare system were formulated and operationalised for subsequent literature search. In a second step, a systematic search was conducted on nationally and internationally available guidelines. Evidencebased suggestions in these guidelines were summarised and methodically assessed using the "German Handbook on Methodological Assessment of treatment guidelines" (http://www.leitlinien. de/leitlinien-grundlagen/leitlinienbewertung/delbi). In a third step, these recommendations were complemented by findings published in systematic reviews of the Cochrane Drugs and Alcohol Library. These reviews provide a comprehensive and systematic overview of the state of the art including quality evaluations. If clinical questions were not answered satisfactorily, a search for further systematic reviews, meta-analyses and singlecase reports was added in a fourth step. A detailed methodological description including the instruments used for the quality checks can be found in the full guideline report (in German, please refer to http://www.awmf.org/leitlinien/detail/ll/076-001.html [6, 8]). For a brief summary, see below. The full report also hosts more than 500 pages of tables (in English) where study synopses are ag-
46

Eur Addict Res 2017;23:45-60 DOI: $10.1159 / 000455841$
Mann/Batra/Fauth-Bühler/Hoch 
Table 1. Level of evidence (LoE) [10]

\begin{tabular}{ll}
\hline LoE & Therapy/prevention, aetiology/harm \\
\hline $1 \mathrm{a}$ & Systematic review (with homogeneity) of randomised controlled trials (RCTs) \\
\hline $1 \mathrm{~b}$ & Individual RCT (with narrow confidence interval) \\
\hline $1 \mathrm{c}$ & $\begin{array}{l}\text { All or none (all patients died before the Rx became available, but some survive on it now; or when some patients died before the Rx } \\
\text { became available, but none die on it now) }\end{array}$ \\
\hline $2 \mathrm{a}$ & Systematic review (with homogeneity) of cohort studies \\
\hline $2 \mathrm{~b}$ & Individual cohort study (including low-quality RCT; e.g., $<80 \%$ follow-up) \\
\hline $2 \mathrm{c}$ & “Outcomes" research; ecological studies \\
\hline $3 \mathrm{a}$ & Systematic review (with homogeneity) of case-control studies \\
\hline $3 \mathrm{~b}$ & Individual case-control study \\
\hline 4 & Case series (and poor quality cohort and case-control studies) \\
\hline 5 & Expert opinion without explicit critical appraisal, or based on physiology, bench research or "first principles" \\
\hline
\end{tabular}

Table 2. Grades of recommendations (modified from [11])

A "Shall" recommendation: at least one randomised controlled study of good quality and consistence, which directly refers to the respective recommendation and which was not extrapolated (evidence level 1a, 1b, 1c)

B "Should" recommendation: well-implemented clinical (non-randomised) study, directly referring to the recommendation (evidence levels $2 \mathrm{a}, 2 \mathrm{~b}, 2 \mathrm{c}$ ) or extrapolation of evidence level 1 , if reference to specific questioning is lacking

O "Can" or "Open” recommendation: evidence categories 3, 4, and 5. Reports of groups of experts or experts' opinion and/or clinical experience of acknowledged authorities or extrapolation of evidence levels $2 a, 2 b$, or $2 c$. This categorisation indicates that directly applicable clinical studies of good quality did not exist or were not available

CCP "Clinical consent point": recommended as good clinical practice ("Good Clinical Practice Point") in consent and due to clinical experience of members of the guideline group as standard in the treatment, for which no experimental scientific exploration is possible or is intended

gregated (look for "Tabellenband” in http://www.awmf.org/fileadmin/user_upload/Leitlinien/076_D_Ges_fuer_Suchtforschung_ und_Suchttherapie/076-001e_S3_Alkohol_2015-01.pdf).

\section{Evaluation of Evidence}

Systematic reviews, meta-analyses and single studies were methodologically evaluated using the checklists of the "Scottish Intercollegiate Guidelines Network [9]." Based on the type and methodological quality of the study, a level of evidence was given (Table 1) and the study details summarised for the guideline report (for link see above). The levels of evidence served as a basis for the grading of recommendations (Table 2; Fig. 1). Further considerations (ethical, clinical, ...) could be considered for grading as well, and may have led to the up- or downgrading of a recommendation by one level.

\section{Structured Way of Finding Consent}

All key recommendations and grades of recommendations were drafted by members of 7 working groups which dealt with specific areas. Subsequently, they were carefully checked by the steering committee composed of seven elected members. In a next step, six consensus meetings (of 1-3 days duration) took place where all recommendations were presented and discussed, and the group of delegates voted on all recommendations. The principles of voting are defined by the German Association of Scientific Medical Associations (AWMF). Recommendations are not only based on evidence of effectiveness (and side effects), but also on other points such as ethical considerations, personal experiences of delegates including patients and relatives and alike (Fig. 1). Recommendations are accepted if only $75 \%$ of all delegates agree to wording and recommendation grades. In this case, the result is called a "consensus". For a "strong consensus" $95 \%$ of participants had to agree. The voting was open.

Delegates for the consensus group had been nominated by 46 specialist societies from Austria, Switzerland and Germany. To avoid systematic bias, an external moderator from the AWMF steered the consensus meetings. To further assure agreement on the guideline recommendations, all documents were sent for final approval to the boards of the participating scientific societies. As the literature continues to expand, the guidelines will be updated every 5 years. 


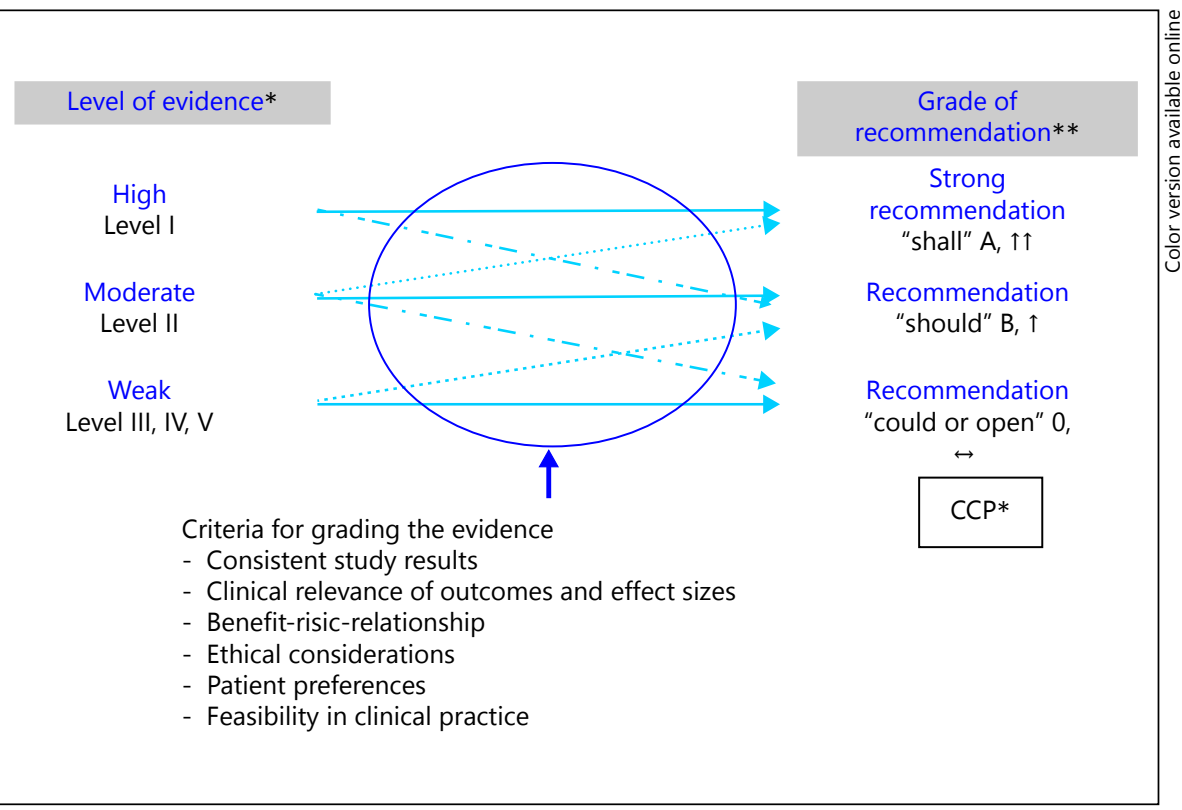

Fig. 1. From level of evidence to grade of recommendation (modified from [12]).

* According to GRADE [13] and Oxford Centre of Evidence-based Medicine [10]. ** According to the German National Guidelines Program. For negative recommendations, the term "not" is added and the same symbols are used.

\section{Potential Conflicts of Interest}

Delegates were asked to declare any interests that could constitute an apparent or potential conflict of interest with respect to his or her involvement in the project. This was repeated on an annual basis (time frame: last 3 years). Experts had to indicate (a) direct financial interests (e.g., reimbursements from companies and organisations working in the healthcare industry, pharmaceutical companies, counselling and non-profit treatment institutions, pensions or healthcare insurances); (b) indirect relations to companies with financial interests in this area (e.g., activities for a lobby group of treatment facilities); (c) immaterial interests regarding academic, subject-specific, political and personal opinions, norms or personal relationships with other participants, which could potentially bias their decision. A member of the steering committee was responsible for double-checking whether the disclosure questions had been assessed. The declaration of interests and decisions on how to proceed with them, were discussed and decided in the consensus meeting. As a result, delegates were allowed to vote only in matters where no conflict of interest existed. This process was double-checked by the moderator of AWMF and the steering committee.

\section{Financing the Guideline}

The guideline "Screening, Diagnosis and Treatment of Alcohol Use Disorders" was developed together with the guideline "Screening, Diagnosis and Treatment of Tobacco-related Disorders" (see Batra et al. [14] for English version). Both guidelines were prepared under the auspices of the German Society for Psychiatry, Psychotherapy and Psychosomatics (Deutsche Gesellschaft für Psychiatrie, Psychotherapie, Psychosomatik und Nervenheilkunde) and the German Society for Addiction Research and Addiction Therapy (Deutsche Gesellschaft für Suchtforschung und Suchttherapie). Fifteen scientific specialist societies, professional associations and organisations were involved in financing the guidelines. The costs for both guidelines together amounted to approximately 400,000€ (excluding expenses of the societies for sending delegates; please refer to the German version for details [6]).

\section{Results}

The essential results of the process are given in this paper. However, recommendations on the impact of psychiatric comorbidities on screening, diagnostics and treatment of AUD patients were published in a separate paper (Preuss et al. [15], English version under review).

\section{Screening and Diagnostics of Intoxication, Harmful Alcohol Consumption and Alcohol Dependence}

AUD are frequent but often remain undiagnosed. State-dependent markers can be used alone or in combination with questionnaires for early screening, precise diagnostics, estimation of disease severity, documentation of disease course and therapy. A state-dependent marker is defined as a characteristic of the clinical status, appropriate for monitoring the disease course and outcome.

From a public health perspective, screenings are recommended in patients aged 14-70 years starting with the initial contact. Screenings should be repeated every 1-2 years.

In this paper, the term "acute" is used when alcohol intake was within the last hours or days. The term "chronic" is used when alcohol was consumed within the 
Table 3. Screening and diagnostics of intoxication, harmful alcohol consumption and alcohol dependence (references marked with $S$ can be found in the suppl. material)

Recommendations $\quad$ Grades

For screening of at-risk alcohol consumption, harmful use or alcohol dependence, the alcohol use disorders identification

A test (AUDIT) shall be applied

Level of evidence (LoE): 1a

References: (S2, S12, S18)

If the AUDIT is too complex, the short version of the AUDIT, the AUDIT-C, should be applied

LoE: not applicable (n.a.)

References: (S2, S3, S7, S9, S21)

For screening purposes, AUDIT or AUDIT-C should be offered to all patients in all settings

LoE: n.a.

References: (S12, S18)

As indicators for acute alcohol consumption, state markers (ethanol) in tidal air and in the blood, ethyl-glucuronide (EtG) and ethyl-sulfate in the urine in different contexts (general practitioners,

in-patient admission, emergency admission, pre-operative screening and intensive care unit) shall be applied

LoE: $1 b$

References: (S5, S6, S10, S11, S12)

As indicators for chronic alcohol consumption, appropriate state markers (EtG in the hair and phosphatidylethanol in the blood) in $\mathrm{B}$ different contexts (in-patient admission, emergency admission, pre-operative screening, intensive care unit) should be applied

LoE: $1 b$

References: (S1, S13, S15, S16, S17, S19, S28)

If chronic alcohol consumption should be attested, a combination of indirect markers (e.g., gamma-glutamyltransferase and mean cell A volume and carbohydrate-deficient-transferrin, antilla index, alc index) shall be applied to increase sensitivity and specificity in different contexts (family practice, in-patient admission, emergency admission, pre-operative screening, intensive care unit)

LoE: $1 \mathrm{a}$

References: (S8, S14, S20, S24, S25)

For a screening on chronic alcohol consumption, the AUDIT and a combination of indirect state markers shall be applied

LoE: $1 b$

References: (S4, S14, S28)

Methods for assessing alcohol consumption should include procedures that allow specifying a quantity-frequency-index CCP (separate questions on quantity and frequency) and frequency and quantity of increased alcohol consumption. Alternatively, drinking assessment methods that allow obtaining estimates of the quantity of daily drinking by using retrospective estimates of daily drinking over a specified time period ("timeline-follow-back")

LoE: n.a.

References: (S13, S22, S23)

To diagnose alcohol misuse or alcohol dependence, validated instruments should be used that ask for relevant criteria outlined in the International Classification of Diseases

LoE: n.a.

References: (S26, S27)

last weeks or months (Tables 3, 4; references in online suppl. material; for all online suppl. material, see www. karger.com/doi/10.1159/000455841).

\section{Comorbid Psychiatric Diseases}

Alcohol consumption can lead to various mental comorbidities. Inter-individual differences exist regarding the disease type and severity. In addition to the diagnostics of AUD, the precise diagnostics of comorbid mental disorders is important (Table 5; references in online suppl.). This chapter was published in German in a separate paper [15], English version under review.

\section{Age- and Gender-Specific Populations}

For the treatment of AUD in age- and gender-specific populations, different treatment recommendations may apply. For example, with respect to children and adolescents, developmental perspectives and fam- 
Table 4. Somatic complications of alcohol use disorders and comorbidities (references marked with S can be found in the suppl. material)

Recommendations

In patients with alcohol-associated somatic diseases, diagnostic assessment for possible alcohol-related liver diseases should be carried out

Level of evidence (LoE): 2

References: (S2, S3, S5)

Patients diagnosed with liver diseases should be examined for alcohol-related disorders

LoE: n.a.

References: (S3, S9)

In cases of alcohol-related liver diseases, abstinence from alcohol is to be aimed at

LoE: $1 b$

References: (S1, S3, S4, S8)

The extent and - if applicable - the progression of fibrosis shall primarily be examined by non-invasive methods (elastography); in certain cases by liver biopsy

LoE: $1 b$

References: (S6)

In cases of alcohol-induced pancreatitis, not only the inflammation of the organ and its complications, but also the underlying alcohol-related disorder should be treated

LoE: n.a.

References: (S7)

In cases of chronic pancreatitis, alcohol consumption shall strictly be avoided

LoE: $2 b$

References: (S7)

Table 5. Concomitant tobacco use (references marked with $S$ can be found in the suppl. material)

\begin{tabular}{l}
\hline Recommendations \\
\hline $\begin{array}{l}\text { Patients with alcohol use disorders and concomitant tobacco use should be offered consultation and support for } \\
\text { smoking cessation }\end{array}$ \\
Level of evidence (LoE): n.a. \\
References: (S4, S7, S16, S17, S18) \\
\hline Patients with alcohol use disorders and concomitant tobacco use shall be offered the same therapeutic interventions \\
for smoking cessation as smokers without alcohol use disorders \\
LoE: 1 b \\
References: (S1, S2, S3, S6, S8, S9, S10, S11, S12, S15) \\
The time point for supporting smoking cessation in patients with alcohol use disorders should be individually \\
coordinated with patients. Thereby, progress in the therapy of alcohol dependence should be taken into \\
consideration \\
LoE: 1 b \\
References: (S5, S8, S9, S13, S13, S15)
\end{tabular}

ily situations should be taken into consideration (Table 6; references in online suppl.). As in other parts of the guidelines, some of the points made in this context are based on clinical experience rather than on scientifically derived evidence. In elderly people, metabolic changes and increased rates of somatic comorbidities and cognitive disabilities need to be taken into consideration (Table 8). For women, and in particular pregnant women, interventions and treatment settings should be tailored to their specific needs (Table 7; references in online suppl.). 
Table 6. Children and adolescents (references marked with S can be found in the suppl. material)

Recommendations

Motivational interviewing (MI) shall be offered to adolescents after alcohol intoxication for short-term reduction of alcohol

A consumption and risky alcohol consumption. MI is not effective regarding long-term reduction of alcohol consumption

Level of evidence (LoE): 1a

References: (S2, S16, S21, S24, S26, S29)

Brief interventions can be offered to adolescents with alcohol use disorders

$\mathrm{O}$

LoE: 5

References: (S24)

Cognitive behavioural therapy shall be offered for the treatment of children and adolescents with alcohol use disorders

LoE: 1 a

References: (S3, S4, S6, S10, S11, S12, S14, S18, S20, S25, S27, S28)

Multi-systemic therapy (MST), short-term family therapy, functional family therapy, and resource-oriented family

$\mathrm{O}$

therapy can be offered for the treatment of children and adolescents with alcohol use disorders

LoE: 1a

References: (S1, S4, S5, S7, S13, S14, S20, S21, S23, S25)

Multi-dimensional family therapy should be offered for the treatment of children and adolescents with alcohol use disorders

LoE: $1 \mathrm{a}$

References: (S1, S4, S14, S15, S20, S21, S25)

Integrative family and cognitive behavioural therapy should be offered for the treatment of children and adolescents with alcohol use disorders

LoE: 1a

References: (S1, S4, S13, S20, S21, S25)

Family members shall be included in the treatment of children and adolescents with alcohol use disorders

LoE: la

References: (S1, S21, S22, S28, S30)

In-patient therapies should be offered to children and adolescents with alcohol withdrawal syndrome. Somatic symptoms

CCP

should be considered and psychosocial support offered

LoE: n.a.

References: (S19)

Educational support for families of children and adolescents with alcohol use disorders can be offered as part of the treatment plan CCP

LoE: n.a.

References: (S7, S21)

During in-patient treatment, children and adolescents with alcohol use disorders should have the possibility to attend clinic

CCP

schools

LoE: n.a.

Regarding relapse prevention with acamprosate or naltrexone, no treatment recommendations can be given for adolescents with alcohol use disorders

LoE: n.a.

References: (S18, S20)

In cases of indication for methylphenidate, treatment of ADHD administration should be doubled-checked if alcohol use disorders coexist. Medication should be carefully planned and monitored

LoE: n.a.

The setting for the treatment of children and adolescents with alcohol use disorders should be chosen based on the following questions:

- How strong is the need for a safe environment?

- How strong is the motivation of the adolescent and her/his family to actively participate in the treatment?

- How strong is the need for structure and clear boundaries?

- Are there any additional medical or psychiatric symptoms and related risks?

- Are specific treatment settings for adolescents available?

- Are there any preferences for treatment in certain settings and are there treatment failures in the past in less

LoE: 4 restrictive/intensive settings?

References: (S18) 
Table 7. Pregnant women (references marked with $\mathrm{S}$ can be found in the suppl. material)

Recommendations

Pregnant women with alcohol use disorders should be offered a brief intervention

CCP

Level of evidence (LoE): n.a.

References: (S8, S26, S28)

Pregnant women with alcohol use disorders should be offered psychotherapeutic interventions

CCP

LoE: n.a.

Pregnant women with alcohol use disorders should be offered mitigation measures and psychosocial interventions

CCP

LoE: n.a.

References: (S8, S20)

Pregnant women with alcohol use disorders can be offered house calls by members with specialized education in

$\mathrm{O}$ jobs in the social and/or healthcare field

LoE: $2 b$

References: (S7, S27)

Women with alcohol use disorders can be offered treatment in a setting for women only

$\mathrm{O}$

LoE: $1 b$

References: (S9, S10, S17, S24)

Table 8. Elderly people (references marked with S can be found in the suppl. material)

Recommendations $\quad$ Grades

Empirically established treatments for younger adults (psychotherapeutic, psychosocial and pharmacotherapeutic) CCP should also be offered for elderly people with alcohol use disorders

Level of evidence (LoE): n.a.

Somatic and psychiatric comorbidities should be considered when planning and implementing interventions and treatment for elderly people with alcohol use disorders

LoE: n.a.

For the use of medication to support detoxification and for harm reduction in elderly people with alcohol use disorders, please see chapter "Post-Acute Treatment for Dependent Individuals after Detoxification"

Elderly people with alcohol use disorders can be offered a low-threshold in-patient treatment

LoE: 5

References: (S5)

Elderly people with alcohol use disorders shall be asked for changes in alcohol consumption in primary care settings

Therapeutic interventions aimed at reducing alcohol intake should be offered

LoE: $1 b$

References: (S1, S2, S3, S4, S6, S7, S8)

\section{Acute Treatment of Harmful Alcohol Use and Alcohol Dependence}

\section{Brief Interventions}

Brief interventions can be applied to motivate individuals with problematic alcohol consumption in nonspecialised settings to reduce their alcohol intake or to achieve abstinence. Brief interventions are defined as interventions with a maximum of 5 sessions, each lasting not more than 60 minutes. The interventions are targeted to reduce alcohol consumption and alcohol-associated problems. Therefore, an integrated approach is used combining: (a) personalised feedback, (b) individual definition of goals, and (c) concrete advice. Brief interventions can be provided in an electronic format. In addition, they can be supplemented by information material in written form or provided in a computer-assisted format. 
Table 9. Brief interventions (references marked with $S$ can be found in the suppl. material)

Recommendations

Brief interventions are effective in reducing problematic alcohol consumption and shall be implemented

Level of evidence (LoE): 1a

References: (S1, S9, S19, S32, S34, S39, S43, S46)

The efficacy of brief interventions in reducing alcohol consumption has been best shown for individuals with

at-risk alcohol intake. Therefore, brief interventions shall be offered to this group of patients

LoE: 1a

References: (S1, S9, S19, S32, S34, S39, S43, S46)

Brief interventions can reduce binge drinking. Brief interventions should, therefore, be offered to this group

LoE: 1a

References: (S7, S8, S11, S12, S14, S16, S17, S18, S20, S22, S28, S29, S30, S31, S36, S37, S47, S48, S51, S56, S57)

Evidence for the efficacy of brief interventions to reduce alcohol consumption in alcohol-dependent individuals is contradictory and not completely supported by literature. Brief interventions may be offered to alcohol-dependent patients

LoE: 1a

References: (S6, S10, S13, S15, S21, S23, S26, S32, S38, S42, S45, S52, S53, S54, S55)

The proof of efficacy for brief interventions does not differ between men and women. Therefore, they shall be offered

independent of gender

LoE: 1a

References: (S4, S5, S9, S32, S33, S34, S50, S57)

Brief interventions should be offered to elderly people with alcohol-related problems (older than 65 years)

LoE: n.a.

References: (S24, S25, S27, S41, S44, S49)

Few studies have assessed individuals with alcohol use disorders and comorbid disorders. As these studies found brief interventions to be successful, this treatment should also be offered to patients that suffer from comorbid conditions

LoE: n.a.

References: (S2, S3, S35)

In primary healthcare, brief interventions shall be offered for reducing problematic alcohol consumption

LoE: 1a

References: (S9, S32, S34, S40)

No adverse effects are known from brief interventions. Therefore, they can be implemented

CCP

LoE: n.a.

References: (S9, S32, S46)

Brief interventions have been studied particularly in primary healthcare. The best evidence exists for primary outpatient care in patients with harmful alcohol use. The evidence for patients in general hospitals is still insufficient. Compared to general hospitals, brief interventions in work places are rarely studied (Table 9; references in online suppl.).

\section{Detoxification and "Qualified Withdrawal Treatment"}

In German language, different terminologies exist for alcohol withdrawal treatment. While 'detoxification' is limited to the treatment of somatic consequences resulting from withdrawal of alcohol consumption irrespective of the underlying diagnosis or severity, a "qualified with- drawal treatment" (definition below) focuses on alcohol dependence. The term "withdrawal treatment" is used to describe both alcohol detoxification and qualified withdrawal.

Physical detoxification includes treatment of alcohol intoxication with neuro-psychiatric deficits and/or alcohol-related withdrawal symptoms. The main goal is to maintain vital functions and to avoid complications (e.g., epileptic seizures or delirium tremens) and reduction/relief of withdrawal effects.

QWT is an acute treatment beyond physical detoxification. Treatment of intoxication and withdrawal symptoms and diagnostics and treatment of mental and somatic comorbidities and secondary diseases are done si- 
Table 10. Detoxification and "qualified withdrawal treatment (QWT)" (references marked with S can be found in the suppl. material)

Recommendations

Alcohol use disorders should be treated

CCP

Level of evidence (LoE): n.a.

For patients with a risk for the development of withdrawal symptoms or an individual risk for withdrawal complications such as seizures or delirious symptoms, withdrawal treatment should be conducted

LoE: n.a.

Symptom-oriented treatment of alcohol withdrawal should be offered instead of using a fixed dosage schema, provided continuous assessment and symptom control can be guaranteed right from the beginning and during the course of the treatment, and the staff is adequately trained in the assessment and monitoring of alcohol withdrawal, for example, by applying standardised assessment instruments LoE: n.a.

In-patient treatment with physical detoxification or qualified detoxification treatment shall be offered in case of alcohol-associated withdrawal seizures and/or withdrawal delirium and/or health-related or psychosocial conditions, under which alcohol abstinence in outpatient settings does not seem achievable

LoE: 2

References: (S5, S11, S27)

In-patient treatment with physical detoxification or qualified detoxification treatment should be offered for alcohol-dependent patients and patients with harmful alcohol use, if at least one of the following criteria is met: severe withdrawal symptoms (to be expected), severe and multiple somatic or mental comorbidities or secondary diseases, suicidality, lack of social support, failure of outpatient detoxification LoE: n.a.

Outpatient detoxification treatment (physical detoxification or qualified detoxification treatment) can be offered, if no major withdrawal symptoms or complications are expected, and high adherence and a supportive social environment exist LoE: n.a.

Outpatient detoxification should only be offered by physicians with sufficient knowledge in alcohol detoxification and an opportunity to perform continuous clinical controls and the option of an emergency service that can be reached $24 \mathrm{~h}$ a day

LoE: 2

References: (S6, S11, S27)

Duration of treatment should be individually adapted to the severity of withdrawal symptoms and to physical and mental comorbidities and secondary diseases

LoE: n.a.

Alcohol-dependent patients who do not want to undergo detoxification should be informed about risks due to non-supervised sudden reduction in alcohol consumption or drink stop, such as withdrawal complications and their treatment possibilities LoE: n.a.

As physical detoxification is not sufficient for treating alcohol dependence, additional help from addiction medical services should be arranged

LoE: n.a.

A QWT should be offered instead of solely physical detoxification

LoE: n.a.

A QWT should be offered in case the patient is ambivalent with respect to subsequent treatment procedures

LoE: n.a.

A QWT should generally last 21 days. Considering the severity of withdrawal symptoms, physical and mental comorbidities which accompany or result from alcohol use disorders, individual treatment duration may vary LoE: n.a.

multaneously. Essential for a QWT are psycho- and socio-therapeutic elements that support the willingness to change, the competence to change and the stabilisation of abstinence. The motivation to seek further support should be increased and corresponding contacts into the regional help system established (e.g., self-help groups, psychotherapy, social work) within the scope of
QWT. When indicated, options for further treatment are arranged, for example, social or medical rehabilitation (Table 10; references in online suppl.).

\section{Pharmacotherapy for Alcohol Detoxification}

If alcohol-dependent individuals stop drinking, clinically relevant and often dangerous withdrawal symptoms 
can be observed. The type and degree of withdrawal symptoms can vary from person to person. Pharmacological treatment can lead to suppression of withdrawal symptoms, prevent acute complications that may be lifethreatening and reduce or prevent long-term complications (Table 11; references in online suppl.).

\section{Post-Acute Treatment for Dependent Individuals after Detoxification}

Post-acute treatment can be offered as an outpatient, full-day outpatient or in-patient weaning (rehabilitation) treatment and pharmacological relapse prevention or other forms.

These comprise institutions for chronically impaired patients, in particular socio-therapeutic institutions for chronic alcohol-dependent individuals with multi-morbid conditions and low-threshold approaches, counselling services and interventions that enhance the possibilities to get a job.

Post-acute treatment is often part of medical rehabilitation to abstain from alcohol. The aim is to maintain, improve or reinstate the performance and functions of the alcohol-dependent individual and to support participation in work-life and society. In Germany, medical rehabilitation for most alcohol-dependent individuals is conducted on behalf of statutory pension insurance with the aims outlined above. The rehabilitation institutions require that the addicted individual stays abstinent or is at least able to minimise relapse regarding frequency, duration and severity (Table 12; references in online suppl.).

\section{Psychosocial Interventions of AUD}

Psychosocial interventions are important and effective in almost every stage of the addiction process. This holds true, for example, for interventions to improve motivation in general medicine, in QWT or as part of a more complex withdrawal treatment and as a stand-alone outpatient intervention in the post-acute phase. The most compelling evidence exists for Motivational Enhancement Therapy [16].

Below we refer primarily to psychosocial and psychotherapeutic interventions which are generally used in German-speaking countries (Table 13; references in online suppl.).A more detailed description of evidence-based psychotherapeutic approaches and treatment recommendations can be found in the long version of the guideline. Proofs of efficacy exist for common psychotherapeutic approaches. However, none of the studies systematically reported any side effects. Therefore, we recommend conducting further studies in which side effects are listed systematically, similar to pharmacotherapy studies.

\section{Pharmacotherapy in Post-Acute Treatment}

In the NICE guidelines (2011) [17], the effectiveness of different medications used for the treatment of alcoholdependent patients was systematically analysed. For the following chapter, we evaluated and adopted the NICE recommendations where appropriate. Nalmefene studies leading to approval by the European Medicines Agency were published from 2013 onwards, which was after our own systematic search was closed (end of 2012). Following the AWMF rules, recommendations for nalmefene treatment were not part of the systematic review and had to be graded as CCP. Disulfiram which has shown efficacy in several studies was not analysed because the manufacturer removed it from the German market.

Overall, some of the recommendations here (and elsewhere) may not be completely in line with meta-analyses and other sources of gathered information. In these cases and after weighing all arguments, a majority of members of the consensus felt that a divergent recommendation was justified (Table 14; references in online suppl.).

\section{Members of "the Guideline Group"}

Klaus Amann, Julia Arens, Anil Batra*, Martin Beutel, Oliver Bilke-Hentsch, Gallus Bischof, Udo Bonnet, Gerhard Bühringer*, Ralf Demmel, Heribert Fleischmann, Jennis Freyer-Adam, Wilma Funke, Dieter Geyer, Euphrosyne Gouzoulis-Mayfrank, Arthur Günthner, Ursula Havemann-Reinecke, Derik Hermann, Eva Hoch*, Bettina Jäpel, Michael Klein*, Andreas Koch, Joachim Köhler, Georg Kremer, Gerhard Längle, Nikolaus Lange, Bodo Lieb, Johannes Lindenmeyer, Karl Mann*, Peter Missel, Tim Neumann, Kay-Uwe Petersen*, Ulrich W. Preuß, Jens Reimer*, Olaf Reis, Gerhard Reymann*, Monika Ridinger, Hans-Jürgen Rumpf, PeterMichael Sack, Ingo Schäfer, Martin Schäfer, Norbert Scherbaum, Welf Schröder, Manfred Singer, Michael Soyka, Claudia Spies, Julian Stappenbeck, Rainer Thomasius, Natascha Thon, Clemens Veltrup, Irmgard Vogt, Tillmann Weber, Georg Weil, Volker Weissinger, Bernd Wessel, Arnold Wieczorek, Klaudia Winkler, Nadja Wirth, Norbert Wodarz, Dirk Wolter, Friedrich M. Wurst.

* Member of the steering committee

\section{Links}

http://www.awmf.org/leitlinien/detail/ll/076-001.html. http://www.awmf.org/uploads/tx_szleitlinien/076-001m_S3Leitlinie_Alkohol_2015-02_02.pdf.

http://www.awmf.org/fileadmin/user_upload/ Leitlinien/076_D_Ges_fuer_Suchtforschung_und_ Suchttherapie/076-001e_S3_Alkohol_2015-01.pdf. 
Table 11. Pharmacotherapy for alcohol detoxification (references marked with $S$ can be found in the suppl. material)

Recommendations

Medication-supported alcohol detoxification treatment is superior to non-treatment with respect to severity of A occurring withdrawal symptoms and frequency of withdrawal complications. Therefore, pharmacotherapy for the alcohol withdrawal syndrome shall be applied considering the severity of withdrawal symptoms and withdrawal complications Level of evidence (LoE): 1a

References: (S3, S5, S11, S20, S27, S28)

Severe and moderate alcohol withdrawal symptoms shall be treated pharmacologically

LoE: $1 \mathrm{a}$

References: (S3, S5, S11, S20, S27)

Minor alcohol withdrawal symptoms can be treated pharmacologically

LoE: 1a

References: (S1)

Benzodiazepines effectively reduce the severity and frequency of alcohol withdrawal symptoms, and the frequency of severe withdrawal complications such as delirium and seizures

Benzodiazepines shall be used for the treatment of the alcohol withdrawal syndrome

LoE: 1a

References: (S3, S4, S20, S27, S28)

For the treatment of delirious syndromes with hallucinations, delirium or agitation, benzodiazepines should be combined B with antipsychotics (especially butyrophenones such as haloperidole)

LoE: 4

References: (S1, S5, S10, S20)

Clomethiazole effectively reduces the severity and frequency of alcohol withdrawal symptoms and the frequency of severe withdrawal complications and seizures

Clomethiazole should be applied under in-patient conditions for the treatment of alcohol withdrawal syndrome

LoE: 1

References: (S1)

For the treatment of delirious syndromes with hallucinations, delusion symptoms and agitation, clomethiazole

should be combined with anti-psychotics (especially butyrophenones, such as haloperidol)

LoE: 1

References: (S1)

Clomethiazole should not be applied in the outpatient alcohol withdrawal treatment due to its low therapeutic range

and its dependence or abuse potential and should in no case be combined with benzodiazepines. Clomethiazole is not

approved for the outpatient treatment of alcohol withdrawal syndrome

LoE: n.a.

References: (S1)

Anticonvulsives should be applied for the avoidance of alcohol withdrawal seizures

LoE: n.a.

References: (S1)

Carbamazepine, valproic acid, gabapentin und oxcarbazepine can be applied for the treatment of minor to moderate

$\mathrm{O}$ alcohol withdrawal symptoms

LoE: 3

References: (S5, S6, S7, S8, S11, S16, S17, S19, S20, S22, S25, S26, S27, S32)

Neuroleptics (e.g., haloperidol) are recommended for acute alcohol delirium with delusions or hallucinations, but should

be combined with, for example, benzodiazepines or clomethiazole as it has no effect on vegetative withdrawal symptoms

LoE: 2

References: (S1)

Beta-Blocker and clonidine are not suitable as monotherapy of the alcohol withdrawal syndrome, but can be combined with benzodiazepines or clomethiazole for the treatment of symptoms in the autonomous nervous system

LoE: 4

References: (S5, S11, S27) 
Table 11. (continued)

Recommendations

Baclofen should not be applied for the treatment of alcohol withdrawal syndrome according to the current literature

LoE: $1 b$

References: (S2, S14)

GHB should not be applied for the treatment of acute alcohol withdrawal syndrome due to its benefit-risk profile

B

LoE: 1a

References: (S13)

Alcohol (under medical supervision) should not be applied for alcohol withdrawal treatment

CCP

LoE: n.a.

References: (S5, S11, S27)

Medications with pro-convulsive and anticholinergic effects such as low potent neuroleptics and tricyclic

CCP

anti-depressants should be avoided for alcohol detoxification

LoE: n.a.

Tiapride can be applied in combination with anticonvulsives for the treatment of minor to moderate alcohol

$\mathrm{O}$

withdrawal symptoms

LoE: 3

References: (S1, S15, S18, S23, S33, S34)

During alcohol detoxification, thiamine should be administered for the prophylaxis of Wernicke's encephalopathy

CCP

LoE: n.a.

References: (S9, S12, S35)

If parenteral glucose administration is required in individuals with alcohol dependence, it should be combined

CCP with a parenteral thiamine application

LoE: n.a.

References: (S21, S29)

During pregnancy, benzodiazepines should be preferred for alcohol detoxification. Administration should be

done in an in-patient and interdisciplinary setting

LoE: n.a.

In case of a poor general health, elderly patients, limited lung or renal function, application of benzodiazepines with medium-length half-life and low dosage and symptom-oriented administration for the treatment of alcohol withdrawal syndrome according to the severity of withdrawal is recommended. In these cases, anti-epileptics can be applied additionally for withdrawal symptoms as monotherapy or symptom-oriented in combination with e.g., tiapride or clonidine

LoE: n.a.

References: (S1)

For liver disease with limited liver function, benzodiazepines with a shorter half-life and low metabolisation rate in

the liver (e.g., oxazepam, lorazepam) are recommended

LoE: n.a.

References: (S5, S11, S27)

In case of limited liver function, gabapentine or levetiracetam can be used for the treatment of seizures or their prophylaxis due to their lack of hepatotoxicity and renal excretion

LoE: n.a.

References: (S7, S17, S23, S24, S25, S26, S30, S31)

In case of suspected mixed intoxication or multiple substance abuse, drug screening for benzodiazepines and opiates and other drugs in the urine should be done prior to the initiation of a specific pharmacotherapy for the alcohol withdrawal syndrome and/or states of excitation in an in-patient follow-up

LoE: n.a. 
Table 12. Post-acute treatment after in-patient and outpatient detoxification (references marked with $S$ can be found in the suppl. material) Recommendations Grades

Post-acute interventions following the withdrawal phase should be offered as subsequent treatment without time gap. For alcohol dependence, the overall goal should be abstinence

Level of evidence (LoE): n.a.

References: (S1, S2, S5, S7, S11, S14, S21, S23, S26, S29, S31)

Abstinence is the primary therapeutic goal for the alcohol dependence syndrome (ICD10: F10.2). If abstinence

A

is currently not achievable or if harmful or risky consumption exists, harm reduction by reducing alcohol

consumption (quantity, time, and frequency) should be the goal

LoE: 1a

References: (S1, S2, S7, S11, S14, S23, S26, S29)

Comorbidity (psychiatric) should be considered and be treated in the post-acute phase

CCP

LoE: n.a.

References: (S2, S4, S7, S10, S11, S14, S19, S20, S25, S29, S30, S31, S33)

In alcohol-dependence, a complex treatment should be offered which comprises a combination of different interventions. These interventions should be implemented by a multi-professional team

LoE: n.a.

References: (S2, S3, S11, S17, S22, S24)

Duration and intensity of post-acute treatment (incl. withdrawal) should be individually based on severity,

comorbidity, existing psychosocial impairments and their consequences

LoE: n.a.

References: (S7, S8, S11, S21, S30)

Table 13. Psychotherapy of alcohol use disorders (references marked with S can be found in the suppl. material)

\section{Recommendations}

Motivational intervention approaches shall be offered as part of the post-acute treatment phase

Level of evidence (LoE): 1a

References: (S2)

Cognitive behavioural therapy shall be offered as part of the post-acute treatment phase

LoE: 1a

References: (S2)

Contingency management should be offered as part of the post-acute treatment phase

LoE: $2 b$

References: (S2, S16)

Work with relatives shall be offered as part of the post-acute treatment phase

LoE: $1 b$

References: (S2)

Couple therapy shall be offered as part of the post-acute treatment phase

LoE: $1 b$

References: (S2)

Psychodynamic short-term therapy should be offered as part of the post-acute treatment phase

LoE: $1 b$

References: (S2)

Supervised patient groups should be offered as part of the post-acute treatment phase

LoE: $2 b$

References: (S2)

Neurocognitive training can be offered in the post-acute treatment phase

LoE: n.a.

References: (S8, S32)

\section{Grades}

A

A

B

A

A

B

B

CCP 
Table 14. Pharmacotherapy in post-acute treatment (references marked with $S$ can be found in the suppl. material)

Recommendations

Grades

Pharmacotherapy with acamprosate or naltrexone should be offered as part of the patients' overall treatment plan in the post-acute phase outside the in-patient withdrawal treatment

Side effects should be taken into consideration and patients should be informed about possible risks associated with

the medication

Level of evidence (LoE): 1a

References: (S1, S2, S3, S9, S14, S18, S23, S29)

In case other approved treatment options were not successful, a pharmacotherapy with disulfiram can be offered as

$\mathrm{O}$ part of the patients' overall treatment plan in the post-acute phase outside the in-patient withdrawal treatment Side effects should be taken into consideration and patients should be informed about possible risks associated with the medication*

LoE: $1 b$

References: (S2, S14, S15, S29)

If the primary goal is a reduction of alcohol consumption, pharmacotherapy with nalmefene can be offered as part CCP of the patients' overall treatment plan in the post-acute phase outside the in-patient withdrawal treatment Side effects should be taken into consideration and patients should be informed about possible risks associated with the medication

LoE: n.a.

References: (S2, S3, S12, S14, S18, S19, S23, S27, S28, S29)

* Please note that in Germany, disulfiram has been removed from the market (company decision).

\section{Acknowledgement}

We would like to thank the guideline consensus group for sharing their expertise and knowledge. The authors are grateful to Ingrid Weber who kept the whole process afloat for over more than 5 years and who provided the baseline translation for this paper. We further thank Angelika Heimann for helping with manuscripts.

\section{Disclosure Statement}

K.M. received fees for consulting from Abbvie, D\&A Pharma, Lundbeck and Novartis. M.F.-B., E.H., and A.B. have no conflicts of interest.

\section{References}

1 Pabst A, Kraus L, Gomes de Matos E, Piontek D: Substanzkonsum und substanzbezogene Störungen in Deutschland im Jahr 2012. Sucht 2013;59:321-331.

2 Gärtner B, et al: Alkohol - Zahlen und Fakten zum Konsum. Jahrbuch Sucht 2013:3666.

3 Salize HJ, Jacke C, Kief S, Franz M, Mann K: Treating alcoholism reduces financial burden on care-givers and increases quality-adjusted life years. Addiction 2013;108:62-70.

4 Singer MV, Batra A, Mann K (Hrsg): Alkohol und Tabak. Grundlagen und Folgeerkrankungen. Stuttgart und New York, Thieme Verlag, 2011, pp 57-62.

5 Rehm J, Rehm M, Shield KD, Gmel G, Frick $\mathrm{U}$, Mann K: Reduzierung alkoholbedingter Mortalität durch Behandlung der Alkoholabhängigkeit [Decrease in alcohol-attributable mortality by treatment of alcohol depen- dents]. Sucht Zeitschrift für Wissenschaft und Praxis 2014;60:93-105.

6 Arbeitsgemeinschaft der Wissenschaftlichen Medizinischen Fachgesellschaften (AWMF) - Ständige Kommission Leitlinien. AWMF-Regelwert "Leitlinien". 1. Auflage 2012. Verfügbar. http://www.awmf. org/leitlinien/awmf-regelwert.html (August 5, 2016).

7 Hoch E, Batra A, Mann K: Das S3-Leitlinienprogramm für substanzbezogener Störungen. Sucht 2012;2:14-25.

8 Mann K, Hoch E, Batra A, Bonnet U, Günthner A, Reymann G, Soyka M, Wodarz N, Schäfer M: Leitlinienorientierte Behandlung alkoholbezogener Störungen [Guideline-oriented treatment of alcohol-related disorders]. Nervenarzt 2016;87:13-25.

9 Scottish Intercollegiate Guidelines Network (SIGN): The management of harmful drink- ing and alcohol dependence in primary care. http://www.sign.ac.uk/guidelines (August 5, 2015).

10 OCEBM Levels of Evidence Working Group: The Oxford Levels of Evidence 2. Oxford Centre for Evidence-Based Medicine. http:// www.cebm.net/index.aspx?o=5653 (August 10, 2015).

11 Bundesärztekammer (BÄK), Arbeitsgemeinschaft der Wissenschaftlichen Medizinischen Fachgesellschaften (AWMF), Kassenärztliche Bundesvereinigung (KBV): Nationales Programm für Versorgungs-Leitlinien. Methoden-Report, 2014.

12 Arbeitsgemeinschaft der Wissenschaftlichen Medizinischen Fachgesellschaften (AWMF) Ständige Kommission Leitlinien. AWMF-Regelwerk "Leitlinien". 1. Aufl. 2013. http://www. awmf.org/leitlinien/awmf-regelwerk.html (August 10, 2015). 
13 Atkins D, Best D, Briss PA, Eccles M, FalckYtter Y, Flottorp S, Guyatt GH, et al; GRADE Working Group: Grading quality of evidence and strength of recommendations. BMJ 2004; 328:1490-1498.

14 Batra A, Petersen KU, Hoch E, Andreas S, Bartsch G, Gohlke H, Jähne A, Kröger C, Lindinger P, Mühlig S, Neumann T, PötschkeLanger M, Ratje U, Rüther T, Schweizer C, Thürauf N, Ulbricht S, Mann K: S3 Guideline
"Screening, diagnostics, and treatment of harmful and addictive tobacco use". Sucht 2016;62:139-152.

15 Preuss UW, Gouzoulis-Mayfrank E, Havemann-Reinecke U, Schäfer I, Beutel M, Mann KF, Hoch E: Psychische Komorbiditäten bei alkoholbedingten Störungen. Nervenarzt 2016;87:26-34

16 Sellman JD, Sullivan PF, Dore GM, Adamson SJ, MacEwan I: A randomized con- trolled trial of motivational enhancement therapy (MET) for mild to moderate alcohol dependence. J Stud Alcohol 2001;62:389396

17 National Institute for Health and Care Excellence: Alcohol-Use Disorders: Diagnosis, Assessment and Management of Harmful Drinking and Alcohol Dependence (CG 115). National Institute for Health and Clinical Excellence, 2011. 